\title{
Studies of ac hopping conduction at low temperatures
}

\author{
Jeppe C. Dyre \\ Institute of Mathematics and Physics (IMFUFA), Roskilde Universitetscenter, Postbox 260, DK-4000 Roskilde, Denmark
}

(Received 4 October 1993; revised manuscript received 13 December 1993)

\begin{abstract}
A method is presented that makes computer simulations of hopping conduction in symmetric hopping models with thermally activated jump rates possible at arbitrarily low temperatures. The method utilizes the ac Miller-Abrahams electrical equivalent circuit which is systematically reduced by the general star-mesh transformation until one ends up with an admittance matrix referring to the voltage generators. From this matrix the conductivity is easily calculated. Results from simulations of hopping in two dimensions are presented and compared to the predictions of the effective-medium approximation (EMA). Generally there is good agreement, with some deviation at the lowest temperatures. It is shown that, as the temperature goes to zero, the frequency-dependent conductivity in the EMA becomes universal, i.e., independent of the activation energy probability distribution. The computer simulations confirm the existence of universality, although there is no exact agreement between the simulations and the EMA universality prediction.
\end{abstract}

\section{INTRODUCTION}

ac conduction in disordered solids like amorphous semiconductors, ionic conductive glasses, polymers, or metal-cluster compounds show a number of common features. $^{1-8}$ Above a characteristic frequency $\omega_{m}$, the conductivity becomes strongly frequency dependent, varying as an approximate power law with an exponent between 0.7 and 1.0. The ac conductivity is always less temperature dependent than the dc conductivity, and at low temperatures the ac conductivity becomes almost temperature independent. A final ubiquitous observation is the Barton, Nakajima, and Namikawa (BNN) relation $^{9-13}$ which essentially expresses the fact that the characteristic frequency $\omega_{m}$ is proportional to the dc conductivity $\sigma(0)$. In particular, these two quantities always have the same activation energy.

The most thoroughly studied models for ac conduction in disordered solids are the so-called hopping models. ${ }^{14-16}$ A hopping model describes jumps of charge carriers in a stochastic framework. The disorder is usually mimicked by assuming that the transition rates vary randomly according to some probability distribution. Linearized hopping models are amenable to simple analytic treatment. Linearized hopping models, henceforth just referred to as hopping models, describe the motion of noninteracting charge carriers. Thus, one ignores selfexclusion as well as Coulomb interactions.

Recently, the role of Coulomb interactions has come into focus. ${ }^{17,18}$ To include the effects of Coulomb repulsion, a "macroscopic" model was studied by the present author, ${ }^{19,20}$ following previous work by Springett, ${ }^{21}$ Webman et al., ${ }^{22}$ Sinkkonen, ${ }^{23}$ and Fishchuk. ${ }^{24}$ When Maxwell's equations for an inhomogeneous semiconductor are discretized, one arrives at an electrical equivalent circuit with nodes placed on a cubic lattice and links between neighboring nodes consisting of a resistor and a capacitor in parallel. ${ }^{20,22,24}$ The capacitor currents are Maxwell's displacement currents while the resistor currents are the free charge currents. ${ }^{20}$ Computer simulations of this model have shown ${ }^{20}$ that the effective medium approximation (EMA) for random admittance networks $^{25}$ works very well, even at low temperatures where the spread in conductances is very large. In particular, the simulations confirmed the EMA prediction ${ }^{19,20}$ of a universal frequency dependence of the conductivity at low temperatures, independent of the probability distribution of the local conductivity activation energy. If one defines $\widetilde{\sigma}=\sigma(\omega) / \sigma(0)$ and $\widetilde{s}=i \widetilde{\omega}$ where $\widetilde{\omega}$ is a suitable dimensionless frequency, the EMA equation for the universal frequency-dependent conductivity in the macroscopic model is

$$
\widetilde{\sigma} \ln (\widetilde{\sigma})=\widetilde{s} .
$$

Reference 19 gave the first general derivation of Eq. (1) and presented the first computer simulations confirming it. Equation (1), however, appeared in the literature already in 1980 in a study of the hopping model with electrons tunneling between positionally disordered sites. ${ }^{26}$ The recent results for the macroscopic model therefore raise a number of questions. Is the EMA for hopping models as reliable as it is for the macroscopic model? In particular, as the temperature goes to zero, does the EMA predict Eq. (1) as the universal low-temperature frequency dependence of the conductivity even for hopping models with thermally activated jump rates? If this is the case, is the universality confirmed by computer simulations? To answer the last question, a new numerical method must be developed since neither the standard Monte Carlo type method nor, e.g., the Gauss-Seidel relaxation method makes it possible to go to low temperatures where the jump rates vary over several decades (often more than 50 decades).

The present paper introduces a new method for computer simulation of hopping models. The method allows one to go to arbitrarily low temperatures without any computational "slowing down." The method utilizes the Miller-Abrahams equivalent circuit which is systemati- 
cally reduced by eliminating nodes according to a transformation well known from electrical engineering. Before this method is presented in Sec. III, Sec. II briefly reviews the basic equations of symmetric hopping models and the EMA equation for the frequency-dependent conductivity in hopping models. Section IV reports the results of computer simulations in $2 \mathrm{D}$ and compares the simulations to the EMA predictions. In Sec. V, the lowtemperature limit of the EMA is studied. It is shown that Eq. (1) is predicted to be the universal lowtemperature conductivity even for hopping models. Also, Eq. (1) is compared to the results of computer simulations. Finally, Sec. VI contains a discussion.

\section{SYMMETRIC LINEAR HOPPING MODELS}

This section briefly reviews symmetric linear hopping models and their approximate solution in the EMA. Since several reviews have been written on this subject, ${ }^{14-16,27-30}$ only the most necessary background is given here.

For simplicity we consider first hopping in one dimension in thermal equilibrium, i.e., with no external electric field. A model solid is regarded in which the charge carriers can be only at regularly spaced discrete sites. Let $N_{i}$ denote the average number of charge carriers at site $i$. This number changes in time because some charge carriers leave site $i$ and others arrive from the neighboring sites $i-1$ and $i+1$ (only nearest-neighbor jumps are allowed). If $\Gamma(i \rightarrow j)$ denotes the probability per unit time of a jump from site $i$ to site $j=i \pm 1$, the basic equation for the average $N_{i}$ 's is

$$
\begin{aligned}
\frac{d N_{i}}{d t}= & -[\Gamma(i \rightarrow i+1)+\Gamma(i \rightarrow i-1)] N_{i} \\
& +\Gamma(i-1 \rightarrow i) N_{i-1}+\Gamma(i+1 \rightarrow i) N_{i+1} .
\end{aligned}
$$

This equation is a simple example of a master equation. ${ }^{31,32}$

In symmetric hopping models one has symmetric equilibrium jump probabilities,

$$
\Gamma(i \rightarrow j)=\Gamma(j \rightarrow i) \equiv \Gamma_{0}(i, j) \quad(j=i \pm 1) .
$$

Introducing the probability of finding a charge carrier at site $i, P_{i}=N_{i} / N$ where $N$ is the total number of charge carriers, and, when Eq. (3) is taken into account, Eq. (2) becomes

$$
\frac{d P_{i}}{d t}=\Gamma_{0}(i-1, i)\left(P_{i-1}-P_{i}\right)-\Gamma_{0}(i, i+1)\left(P_{i}-P_{i+1}\right)
$$

The stationary solution of Eq. (4) corresponds to all sites being equally probable. Any initial nonhomogeneous distribution of charge carriers will eventually equilibrate through "diffusion" of charge carriers away from densely populated sites.

Equation (4) is linear. This is the mathematical expression of the fact that the equation deals with noninteracting charge carriers. The charge carriers cannot feel each other: neither is Coulomb repulsion taken into account nor is "self-exclusion" (i.e., that there is room for only one charge carrier at each site). In some papers dealing with hopping models, more general nonlinear hopping models are formulated, attempting to take these effects into account. Equation (4) is then arrived at by linearizing the general hopping equation. However, as pointed out by Shklovskii and Efros, this linearization is not exact, but involves uncontrolled approximations. ${ }^{33}$

For systems of tunneling electrons, the transition rates depend exponentially on the tunneling distance (as well as on the difference of the site energies). In the present paper we are concerned with the "classical" case where the jump rates are thermally activated, i.e., where one has

$$
\Gamma_{0}(i, j)=\Gamma_{0} e^{-\beta E_{i, j}} \quad(j=i \pm 1) .
$$

Here $\beta=1 /\left(k_{B} T\right)$, while $E_{i, j}$ is the so-called activation energy - the barrier to be overcome. The prefactor $\Gamma_{0}$ is the "attack frequency" which is usually of order $10^{12} \mathrm{~Hz}$ (a typical phonon frequency). Equation (5) is relevant for ionic conduction and for certain cases of polaronic conduction.

In symmetric hopping models one usually assumes that the jump rates vary randomly, so the model is completely characterized by the activation energy probability distribution $p(E)$. Figure 1 shows an example of the potential for a symmetric hopping model in $1 D$ with thermally activated jumps. At low temperatures the jump rates vary several orders of magnitude, and a charge carrier makes many jumps between pairs of sites separated by low barriers. Via the fluctuation-dissipation theorem this implies that the conductivity depends strongly on frequency.

Before considering the effect of an electric field, we briefly discuss the boundary conditions. The bulk behavior of the model is calculated by letting the volume go to infinity. In any computer simulation, however, only a finite sample is present and one has to specify the boundary conditions. The case of periodic boundary conditions is characterized by requiring $P_{0}=P_{N}$ if the sites are numbered from 0 to $N$. An alternative is the case of blocking electrodes, i.e., to modify Eq. (4) at the end points so that no charge carriers may pass beyond these.

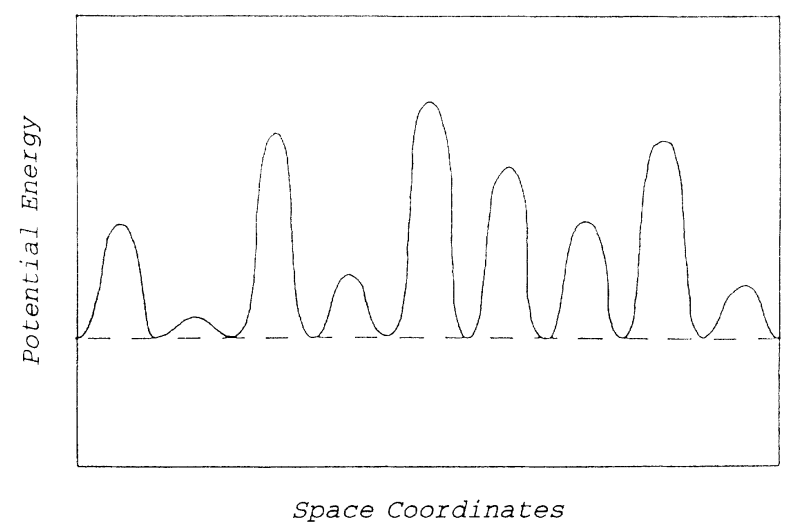

FIG. 1. Potential felt by a charge carrier in a onedimensional symmetric hopping model with thermally activated jump rates. If $\beta$ denotes $1 /\left(k_{B} T\right)$ and $E$ is the energy barrier height, the rate for jumps between two sites is $\Gamma_{0} e^{-\beta E}$. 
A third possible boundary condition is the case of perfectly conducting electrodes. This is arrived at by imposing a fixed probability at the end points:

$$
P_{0}=P_{N}=\text { const . }
$$

Equation (6) corresponds to having no accumulation at the electrodes, allowing completely free passage for the charge carriers. This boundary condition is used below in the computer simulations.

In an external electric field the symmetry of the jump rates is broken, since it becomes more favorable to jump in the direction of the field than opposite to it (assuming the charge $q>0$ ). If the energy barrier is placed symmetrically between the two charge-carrier sites and $a$ denotes the distance between neighboring sites, the jump rates are modified ${ }^{14}$ according to [note that $E$ in Eqs. (7)-(9) below is the electric field and not an energy barrier]

$$
\begin{aligned}
& \Gamma(i \rightarrow i+1, E)=\Gamma_{0}(i, i+1) e^{\beta q a E / 2}, \\
& \Gamma(i+1 \rightarrow i, E)=\Gamma_{0}(i, i+1) e^{-\beta q a E / 2} .
\end{aligned}
$$

In the linear limit (which defines the ordinary conductivity) Eq. (7) is expanded to first order in the electric field:

$$
\begin{aligned}
& \Gamma(i \rightarrow i+1, E)=\Gamma_{0}(i, i+1)\left(1+E \frac{\beta q a}{2}\right), \\
& \Gamma(i+1 \rightarrow i, E)=\Gamma_{0}(i, i+1)\left(1-E \frac{\beta q a}{2}\right) .
\end{aligned}
$$

Consequently, Eq. (2) in terms of the $P_{i}$ 's becomes

$$
\begin{gathered}
\frac{d P_{i}}{d t}=\Gamma_{0}(i-1, i)\left(P_{i-1}-P_{i}\right)-\Gamma_{0}(i, i+1)\left(P_{i}-P_{i+1}\right) \\
+E \frac{\beta q a}{2}\left[\Gamma_{0}(i-1, i)\left(P_{i-1}+P_{i}\right)\right. \\
\left.-\Gamma_{0}(i, i+1)\left(P_{i}+P_{i+1}\right)\right] .
\end{gathered}
$$

In this equation the electric field may depend on time in any arbitrary way.

The complex frequency-dependent conductivity $\sigma(\omega)$ is defined as the ratio between the spatially averaged current density and the electric field in a steady state, where the electric field varies periodically as $e^{i \omega t}$. The Kubo formula ${ }^{34}$ expresses the frequency-dependent conductivity in terms of the current-current time autocorrelation function. For hopping models it is convenient to rewrite the Kubo formula by performing two partial integrations. This leads ${ }^{35}$ to the following expression

$$
\sigma(\omega)=-\frac{n q^{2} \omega^{2}}{2 k_{B} T} \int_{0}^{\infty}\left\langle\Delta x^{2}(t)\right\rangle_{0} e^{-i \omega t} d t .
$$

Here, $n$ is the average charge carrier density, $\left\langle\Delta x^{2}(t)\right\rangle_{0}$ denotes the equilibrium mean-square displacement of a charge carrier in time $t$, and a convergence factor $\lim _{\epsilon \rightarrow 0} e^{-\epsilon t}$ is implicitly understood in the integral.

For a homogeneous system (i.e., with all jump rates equal) the mean-square displacement is determined from the diffusion constant $D_{0}$ via Einstein's equation
$\left\langle\Delta x^{2}(t)\right\rangle_{0}=2 D_{0} t$. As is easy to see, Eq. (10) then gives the frequency-independent conductivity $\sigma=n q^{2} \mu$ where $\mu$ is the mobility which is given by the Nernst-Einstein relation $\mu=D_{0} /\left(k_{B} T\right)$. In the time $t$ a charge carrier in a homogeneous system with equilibrium jump rate $\Gamma$ performs on the average $N=2 \Gamma t$ jumps. Therefore, one has

$$
\left\langle\Delta x^{2}(t)\right\rangle_{0}=N a^{2}=2 \Gamma a^{2} t,
$$

which implies $D_{0}=\Gamma a^{2}$. Combining these equations, one finds for the frequency-independent conductivity of a homogeneous system

$$
\sigma=\frac{n q^{2} a^{2}}{k_{B} T} \Gamma .
$$

For any inhomogeneous system, the high-frequency limit of the conductivity is given by a similar expression. It can be shown ${ }^{36}$ that $\sigma(\infty)$ is given simply by the average jump frequency:

$$
\sigma(\infty)=\frac{n q^{2} a^{2}}{k_{B} T}\langle\Gamma\rangle
$$

It is convenient to redefine the conductivity and absorb the prefactor $n q^{2} a^{2} /\left(k_{B} T\right)$. In this "rationalized" unit system, which will be used henceforth, Eq. (12) simply becomes $\sigma(\infty)=\langle\Gamma\rangle$.

It is also possible to calculate the dc conductivity exactly in one dimension. ${ }^{36}$ Thus, in 1D the high and low frequency limits are in the "rationalized" unit system given by

$$
\begin{aligned}
& \sigma_{1-D}(0)=\left\langle\Gamma^{-1}\right\rangle^{-1}, \\
& \sigma_{1-D}(\infty)=\langle\Gamma\rangle .
\end{aligned}
$$

The inequality $1 \leq\langle\Gamma\rangle\left\langle\Gamma^{-1}\right\rangle$ may be derived from the Cauchy-Schwartz inequality; it implies that $\sigma_{1-D}(0) \leq \sigma_{1-D}(\infty)$. In fact, it can be shown ${ }^{37}$ for any hopping model in any number of dimensions that the real part of the conductivity is an increasing function of the frequency.

Hopping models in $D$ dimensions for $D>1$ are straightforward generalizations of the one-dimensional case. If $P_{s}$ denotes the probability of finding a charge carrier at site s, Eq. (4) is replaced by (for symmetric hopping models)

$$
\frac{d}{d t} P_{\mathrm{s}}=\sum_{\mathbf{s}^{\prime}} \Gamma_{0}\left(\mathrm{~s}, \mathrm{~s}^{\prime}\right)\left(P_{\mathrm{s}^{\prime}}-P_{\mathrm{s}}\right)
$$

In the present paper the sites $s$ are assumed to lie on a simple cubic lattice and the sum is restricted to nearest neighbors. The Kubo formula Eq. (10) is also valid for $D>1$ where the mean-square displacement $\left\langle\Delta x^{2}(t)\right\rangle_{0}$ is in any of the $D$ axis directions. The three abovementioned boundary conditions may also be applied for $D>1$. In an external field, the concepts of blocking or perfectly conducting electrodes make sense only for the sample faces perpendicular to the field; it is natural to apply periodic boundary conditions to all remaining faces.

Even for $D>1$ it is convenient to use the "rationalized" unit system representing the conductivity in terms of an equivalent jump frequency. In this unit system the 
high-frequency limit of the conductivity is for any $D$ given $^{28,37}$ by

$$
\sigma(\infty)=\langle\Gamma\rangle
$$

For the dc conductivity, on the other hand, there is no general analytical expression similar to Eq. (13) in one dimension. However, the temperature dependence of $\sigma(0)$ is known; it is given ${ }^{38-40}$ by

$$
\sigma(0) \propto e^{-\beta E_{c}} \quad(D>1),
$$

where the so-called percolation energy $E_{c}$ in terms of the activation energy probability distribution $p(E)$ and the link percolation threshold $p_{c}$ is given by

$$
\int_{-\infty}^{E_{c}} p(E) d E=p_{c} \text {. }
$$

For $D=2$ one has $p_{c}=\frac{1}{2}$ exactly $^{41}$ while for $D=3$ simulations have shown that $p_{c}=0.2488 .^{42}$

The calculation of the frequency-dependent conductivity in hopping models is a complicated problem. The standard approximation for disordered systems is the coherent potential approximation (CPA). ${ }^{4,44}$ The CPA is a mean-field approximation that gives an estimate of the relevant Green's function that has a number of attractive analyticity properties. For hopping models, the CPA is known as the effective medium approximation (EMA) because it is derived by considering one "link" (i.e., jump frequency) of the lattice as embedded in an "effective medium" mimicking the average surroundings. Several papers ${ }^{15,26,30,45-48}$ derive the EMA equation. If the complex frequency $s=i \omega$ is introduced-referred to below as the "Laplace frequency" - the conductivity $\sigma=\sigma(s)$ in the "rationalized" unit system introduced above is in the EMA given ${ }^{15}$ as the solution of the equation

$$
\left\langle\frac{\Gamma-\sigma}{D \sigma+[1-s \widetilde{G}](\Gamma-\sigma)}\right\rangle_{\Gamma}=0 .
$$

In Eq. (18) the average is over the jump frequency probability distribution and $s \widetilde{G}$ is defined by

$$
s \widetilde{G}=\int_{1-B Z} \frac{d^{D} k}{(2 \pi)^{D}} \frac{s}{s+2 D \sigma[1-p(\mathbf{k})]},
$$

where the integral is over the first Brillouin zone $\left(-\pi<k_{i}<\pi\right)$ and

$$
p(\mathbf{k})=\frac{1}{D} \sum_{i=1}^{D} \cos \left(k_{i}\right) \text {. }
$$

For $s \rightarrow \infty$ one has $s \widetilde{G} \rightarrow 1$. Thus, Eq. (18) implies the correct high-frequency limit Eq. (15). In the limit $s \rightarrow 0$ one has $s \widetilde{G} \rightarrow 0$. Thus, the EMA equation for the dc conductivity is

$$
\left\langle\frac{\Gamma-\sigma(0)}{\Gamma+(D-1) \sigma(0)}\right\rangle_{\Gamma}=0 \text {. }
$$

For $D=1$ Eq. (21) gives the correct result Eq. (13).

To solve Eq. (18) numerically one needs to calculate the quantity $s \widetilde{G}$ of Eq. (19). This is a standard exercise in calculating the diagonal element of the Green's function for a random walk on a cubic lattice or, equivalently, for the quantum-mechanical tight-binding model. ${ }^{49}$ In $1 D$ one finds ${ }^{49,50}$

$$
s \widetilde{G}_{1 \mathrm{D}}=\left(1+\frac{4}{x}\right)^{-1 / 2} \quad(x=s / \sigma) .
$$

In two dimensions one finds ${ }^{49,50}$

$$
s \widetilde{G}_{2 \mathrm{D}}=\frac{2}{\pi} \frac{x}{4+x} K\left(\frac{4}{4+x}\right) \quad(x=s / \sigma),
$$

where $K$ is the complete elliptic integral of the first kind. In three dimensions one finds 49

$$
\begin{aligned}
s \widetilde{G}_{3 \mathrm{D}}= & \frac{x}{2 \pi^{2}} \int_{0}^{\pi} t(\phi) K[t(\phi)] d \phi \\
& \left(t(\phi)=\frac{4}{x+6-2 \cos (\phi)}, x=s / \sigma\right) .
\end{aligned}
$$

Note that Eqs. (22)-(24) all imply $s \widetilde{G} \rightarrow 1$ as $s \rightarrow \infty$, as required by Eq. (19). A rough analytical approximation to Eq. (24) is Hubbard's Green's function ${ }^{49}$

$$
\begin{aligned}
& s \widetilde{G}_{3 \mathrm{D}} \cong \frac{2}{1+6 / x+\sqrt{1+12 / x}} \quad(x=s / \sigma) \\
& \text { III. A METHOD FOR THE NUMERICAL } \\
& \text { EVALUATION OF THE FREQUENCY-DEPENDENT } \\
& \text { CONDUCTIVITY IN HOPPING MODELS }
\end{aligned}
$$

The frequency-dependent conductivity may be evaluated numerically in several ways. In principle, the problem is the straightforward one to solve a large system of linear equations with complex coefficients; from the solution, the conductivity is easily calculated. The GaussSeidel or the Jacobi relaxation methods ${ }^{51}$ are usually applied to such a problem. Unfortunately, they converge much too slowly if the coefficients vary over several orders of magnitude, as is the case when one wants to study hopping at low temperatures. Overrelaxation methods ${ }^{51}$ may be faster, but are still too slow.

An obvious way of evaluating the frequency-dependent conductivity in hopping models is to use an equivalent of a Monte Carlo simulation, simulating the charge-carrier jumps in "real" time. This method works fine at high temperatures, but for $\beta$ larger than about 10 the charge carriers get caught and tend to jump backwards and forwards between two sites without moving away until after thousands or millions of Monte Carlo steps. While this behavior reflects the real physics of low-temperature hopping, the method is clearly very inefficient.

We now proceed to describe an alternative method for evaluating $\sigma(s)$, where a systematic reduction is applied to the ac Miller-Abrahams (ACMA) equivalent circuit. The reduction ends up with a frequency-dependent admittance matrix from which the conductivity is easily calculated. To describe the method we first review the one-dimensional ACMA equivalent circuit, and then show how to reduce the circuit. Finally, the generalization of the method to higher dimensions is discussed. 
To solve Eq. (9) one notes that for small electrical fields the probabilities $P_{i}$ are only slightly different from the average probability $\langle P\rangle: P_{i}=\langle P\rangle+\delta P_{i}$. When substituted into Eq. (9) this gives to first order in $\epsilon$ and $\delta P_{i}$ where $\epsilon=\beta q a\langle P\rangle E$ is a dimensionless measure of the electric field

$$
\begin{aligned}
\frac{d}{d t} \delta P_{i}= & \Gamma_{0}(i-1, i)\left(\delta P_{i-1}-\delta P_{i}\right) \\
& -\Gamma_{0}(i, i+1)\left(\delta P_{i}-\delta P_{i+1}\right) \\
& +\epsilon\left[\Gamma_{0}(i-1, i)-\Gamma_{0}(i, i+1)\right] .
\end{aligned}
$$

Consider now the ACMA electrical circuit shown in Fig. 2(a). The capacitors all have capacity equal to one while the (real) conductance between site $i$ and $i+1$ is $\Gamma_{0}(i, i+1)$. The voltage generators impose the potential drop $-i \epsilon$ from the capacitors to the ground. If the voltage at site $i$ is denoted by $U_{i}$, the Kirchhoff law expressing charge conservation is

$$
\begin{aligned}
\frac{d}{d t}\left(U_{i}+i \epsilon\right)= & \Gamma_{0}(i-1, i)\left(U_{i-1}-U_{i}\right) \\
& -\Gamma_{0}(i, i+1)\left(U_{i}-U_{i+1}\right) .
\end{aligned}
$$

(a)

\section{(b)}
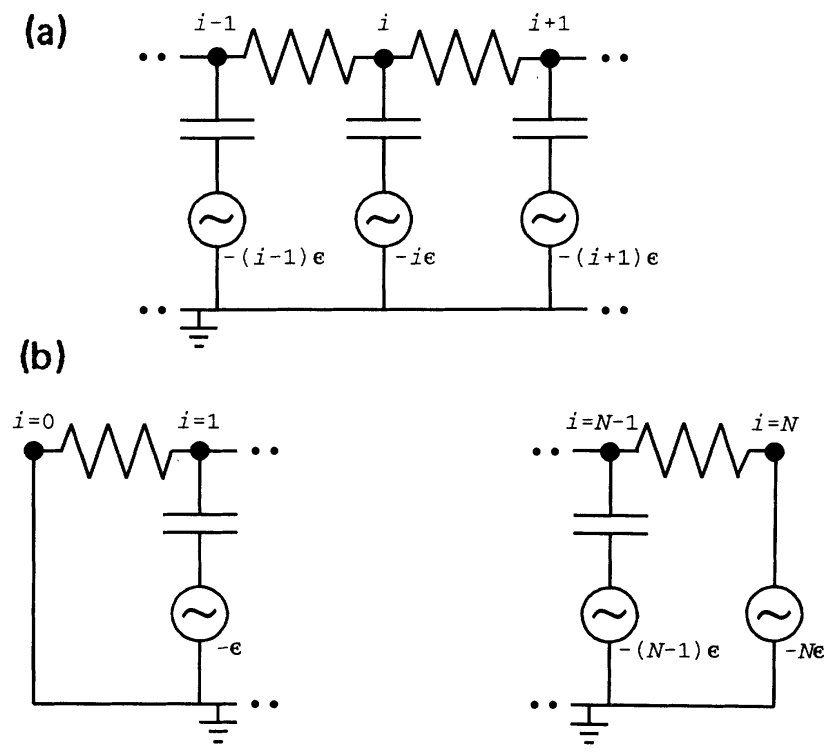

FIG. 2. (a) ac Miller-Abrahams (ACMA) electrical equivalent circuit of a symmetric hopping model in one dimension. All capacitors have unit capacitance while the conductance of the resistor between site $i$ and site $i+1$ is equal to the equilibrium jump frequency $\Gamma_{0}(i, i+1)$. The electric field in the sample, for which $\epsilon$ is a dimensionless measure, is reflected in the voltage generators. The currents in the resistors are equal to the currents in the hopping model [Eq. (30)]. Similar electrical equivalent circuits exist in higher dimensions. Here, the voltage depends only on the coordinate in the direction of the electric field, and thus the capacitors in a plane perpendicular to the field are all connected to the same voltage generator. This fact is crucial for the numerical method for calculating the frequency-dependent conductivity derived in Sec. III. (b) Boundary conditions to the ACMA circuit in one dimension. These boundary conditions correspond to perfectly conducting electrodes [Eq. (6)].
This equation transforms into Eq. (26) if one makes the identification

$$
U_{i}=\delta P_{i}-i \epsilon
$$

Solving Eq. (26) therefore becomes equivalent to solving the ACMA circuit. ${ }^{27,52-54}$ To completely specify the problem the boundaries must be considered. ${ }^{14}$ We use perfectly conducting electrodes for which the boundary conditions are $\delta P_{0}=\delta P_{N}=0$ [Eq. (6)]. For $i=0$ this implies $U_{0}=0$ while for $i=N$ the condition is $U_{N}=-N \epsilon$. These two boundary conditions correspond to the circuit endings without capacitors shown in Fig. 2(b).

Before the ACMA circuit is "solved," let us consider the calculation of the frequency-dependent conductivity from the solution. By definition, $\sigma(s)$ is the ratio between the spatially averaged current and the electric field in a steady periodic situation. If $\langle J\rangle_{\epsilon}$ denotes the spatially averaged current in the field $\epsilon$, we have (where $K$ is a proportionality constant depending on the unit system)

$$
\langle J\rangle_{\epsilon}=K \sum_{i=0}^{N-1}\left[\Gamma(i \rightarrow i+1) P_{i}-\Gamma(i+1 \rightarrow i) P_{i+1}\right] .
$$

To first order in $\epsilon=\beta q a\langle P\rangle E$ this expression via Eqs. (8) and $(28)$ reduces to

$$
\begin{aligned}
\langle J\rangle_{\epsilon} & =K \sum_{i=0}^{N-1} \Gamma_{0}(i, i+1)\left[2 \frac{\epsilon}{2\langle P\rangle}\langle P\rangle+\delta P_{i}-\delta P_{i+1}\right) \\
& =K \sum_{i=0}^{N-1} \Gamma_{0}(i, i+1)\left(U_{i}-U_{i+1}\right)
\end{aligned}
$$

At very high frequencies the capacitors may be ignored and the potential drop across each resistor simply becomes $\epsilon$. In order to reproduce Eq. (13) for the highfrequency conductivity, the general expression for the conductivity in the "rationalized" unit system must therefore be

$$
\sigma=\frac{1}{N} \sum_{i=0}^{N-1} \Gamma_{0}(i, i+1)\left(U_{i}-U_{i+1}\right) \quad(\epsilon=1)
$$

If $I_{R}(i \rightarrow i+1)$ denotes the current in the resistor from site $i$ to site $i+1$ when $\epsilon=1$, one has

$$
\sigma=\frac{1}{N} \sum_{i=0}^{N-1} I_{R}(i \rightarrow i+1) \quad(\epsilon=1) .
$$

In the dc limit the capacitors are completely blocking and only the voltage generator at the site $i=N$ matters [since here there is no capacitor, compare Fig. 2(b)]. The ACMA circuit then effectively reduces to resistances in series and the current is the same in each resistor. This current is determined by the total resistance from $i=0$ to $i=N$. Each resistor has the value $1 / \Gamma_{0}(i, i+1)$ and the total resistance is the sum of all resistors. When the current thus determined is substituted into Eq. (32), one finds the expression given in Eq. (13) for the dc conductivity of a 1D hopping model.

Returning now to the case of an arbitrary frequency (but still in 1D), it is convenient to rewrite Eq. (32) in terms of the current through each voltage generator. If $I_{V}(i)$ denotes the current "upwards" through the $i$ th 
voltage generator towards site $i$, charge conservation implies

$$
\begin{aligned}
& I_{R}(0 \rightarrow 1)=I_{V}(0), \\
& I_{R}(1 \rightarrow 2)=I_{R}(0 \rightarrow 1)+I_{V}(1)=I_{V}(0)+I_{V}(1) ;
\end{aligned}
$$

in general,

$$
I_{R}(i \rightarrow i+1)=I_{V}(0)+\cdots+I_{V}(i) .
$$

When substituted into Eq. (32) this gives

$$
\sigma=\frac{1}{N} \sum_{i=0}^{N}(N-i) I_{V}(i) \quad(\epsilon=1) .
$$

Equation (35) suggests regarding the ACMA circuit as an $N$ port consisting of all the capacitors and the resistors as "internal" elements with "external" nodes that are to be subjected to the potentials $-\epsilon, \ldots,-N \epsilon$ relative to the ground defined by site 0 . Such a circuit is characterized by a (frequency-dependent) symmetric matrix of admittances. This matrix is here defined by

$$
I_{V}(i)=\sum_{j=0}^{N} Y[i, j]\left(U_{i}-U_{j}\right),
$$

where $Y[i, i]$ may be any number. In particular, it is possible from $Y[i, j]$ to calculate the generator currents for $\epsilon=1$,

$$
I_{V}(i)=\sum_{j=0}^{N}(j-i) Y[i, j] .
$$

Substituting this into Eq. (35) one finally arrives at

$$
\sigma=\frac{1}{N} \sum_{i=0}^{N} \sum_{j=0}^{N}(N-i)(j-i) Y[i, j] .
$$

The problem is now reduced to calculating the admittance matrix. This is done by utilizing the general starmesh transformation well known from electrical engineering. ${ }^{55}$ This transformation, which was first used for random resistor networks by Fogelholm, ${ }^{56,57}$ is a prescription of how to remove nodes from a circuit without changing the "external" properties of the circuit. Consider any node in an electrical circuit connected to $m$ other nodes by the admittances $Y_{1}, \ldots, Y_{m}$. This is illustrated in Fig. 3 for the case $m=5$. The central node may be removed by introducing new admittances between all possible pairs of the $m$ neighbor nodes. The new admittance between the neighbor nodes $i$ and $j, Y_{i j}$, is given ${ }^{55}$ by

$$
Y_{i j}=\frac{Y_{i} Y_{j}}{Y_{1}+\cdots+Y_{m}} .
$$

If some of the $m$ neighbor nodes were already connected by an admittance before the transformation, this admittance is increased by the amount given by Eq. (39). What does it actually mean physically that the new circuit is "equivalent" to the old? This means that, for all possible choices of potentials applied to the $m$ neighbor nodes, the same currents run from each of these nodes into the circuit. In this sense, the $m$ neighbor nodes cannot detect any difference before and after the transformation. Once this condition has been specified, it is straightforward to

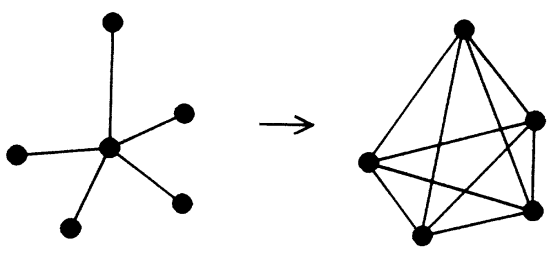

FIG. 3. General star-mesh transformation of an electrical circuit (Ref. 55). In this example the central node is removed. This node is connected to five neighboring nodes by the admittances $Y_{1}, \ldots, Y_{5}$. After the transformation, all possible connections between the five "external" nodes are created by, between the $i$ th and the $j$ th node, introducing the admittance $Y_{i} Y_{j} /\left(Y_{1}+\cdots+Y_{5}\right)$. Physically, the fact that the new circuit is equivalent to the old means that, for any "external" potentials applied to the five nodes, the same currents run into the circuit before and after the transformation. The general star-mesh transformation may be applied to the ACMA circuit of Fig. 2 and its higher-dimensional analogs. When all nodes have been removed one is left with an admittance matrix $Y[i, j]$ which directly determines the frequency-dependent conductivity [Eq. (38) in $1 \mathrm{D}$ or Eq. $(40)$ in $2 \mathrm{D}]$.

derive Eq. (39).

When this transformation is applied to the ACMA circuit each of the "internal" nodes indexed $i=0,1, \ldots, N$ is removed. Eventually, one is left with all possible connections between the "external" nodes. Each connection has an admittance which specifies the corresponding matrix element in the admittance matrix $Y[i, j]$. With this method for evaluating $\sigma(s)$ for a hopping model, the number of calculations that are to be performed is independent of the temperature. A further advantage is that the present method, by proceeding through a number of simple algebraic operations on the circuit admittances without any subtractions, introduces virtually no numerical inaccuracies. Thus, the conductivity is evaluated with a very high precision.

The method is easily generalized to higher dimensions. Considering the case $D=2$, the ACMA circuit is a square lattice here whose nodes are indexed by $(i, k)$ where $i=0, \ldots, N$ and $k=1, \ldots, N$. As in Fig. 2(a) each node on the lattice is connected to the ground via a capacitor and a voltage generator. ${ }^{14,54}$ Neighboring nodes are connected by a resistor whose conductance is the equilibrium jump frequency. The external electric field is assumed to be in the direction of the $x$ axis (indexed by $i$ ). This means that the voltage generators have a voltage equal to $-i \epsilon$, independent of $k$. In effect, there is thus just one voltage generator for each $i$. In the $y$ direction we use periodic boundary conditions so that the point $(i, N+1)$ is identified with the point $(i, 1)$. In the $x$ direction the perfect electrode boundary condition is used. For calculating the conductivity, all nodes $(i, k)$ with $i=1, \ldots, N-1$ are removed according to the recipe of Eq. (39). After the reduction has been performed one ends up with an $(N+1) \times(N+1)$ symmetric admittance matrix $Y[i, j]$, where both indexes refer to the $x$ coordinates. The calculation of the conductivity from the matrix proceeds as in 
Eq. (38), except that a further division by $N$ is necessary to compensate for the fact that each "layer" perpendicular to the $x$ axis has $N$ parallel channels. Introducing an $s$ to remind of the fact that the conductivity is frequency dependent, we thus get

$$
\sigma(s)=\frac{1}{N^{2}} \sum_{i=0}^{N} \sum_{j=0}^{N}(N-i)(j-i) Y[i, j ; s] .
$$

It is not clear in which order the nodes should be removed to have the fastest algorithm. This point is important because the removal of one node introduces several new connections. And the more connections there are to a given node, the more calculations are required for removing it. The nodes should therefore be removed so that as few new connections as possible are created. In the original Fogelholm algorithm in each step one removes the node with fewest connections to its surroundings. ${ }^{57}$ This works well for a system where most neighboring nodes are not connected, as is the case close to the link percolation threshold. In the present case, however, where all neighbors are connected, this procedure becomes very inefficient, because the last nodes to be removed become excessively costly. We found it better to "contain the damage" by removing one column at time (i.e., the nodes with the same index $i$ ). After the first $p$ columns have been removed, one has a situation where the $p+1$ "electrodes" connected to the voltages $0,-1, \ldots,-p$ are all connected to each other. Furthermore, all possible connections exist from these $p+1$ "electrodes" to the $N$ nodes of the $(p+1)$ th column, and all nodes in the $(p+1)$ th column are connected to each other. A further optimization of the algorithm is obtained by, after removing the first $N / 2$ columns, starting from the other end of the circuit by removing columns in decreasing order of the $i$ index.

\section{COMPUTER SIMULATIONS}

The algorithm derived in Sec. III was applied to a study of hopping in two dimensions. At low temperatures large lattices are needed to obtain reasonable statistics. We chose to simulate hopping on a $100 \times 100$ lattice. For this system a standard workstation calculates the conductivity accurately (at one particular frequency) in about $1 \mathrm{~min}$. However, even a $100 \times 100$ lattice is not self-averaging at low temperatures, and it was necessary
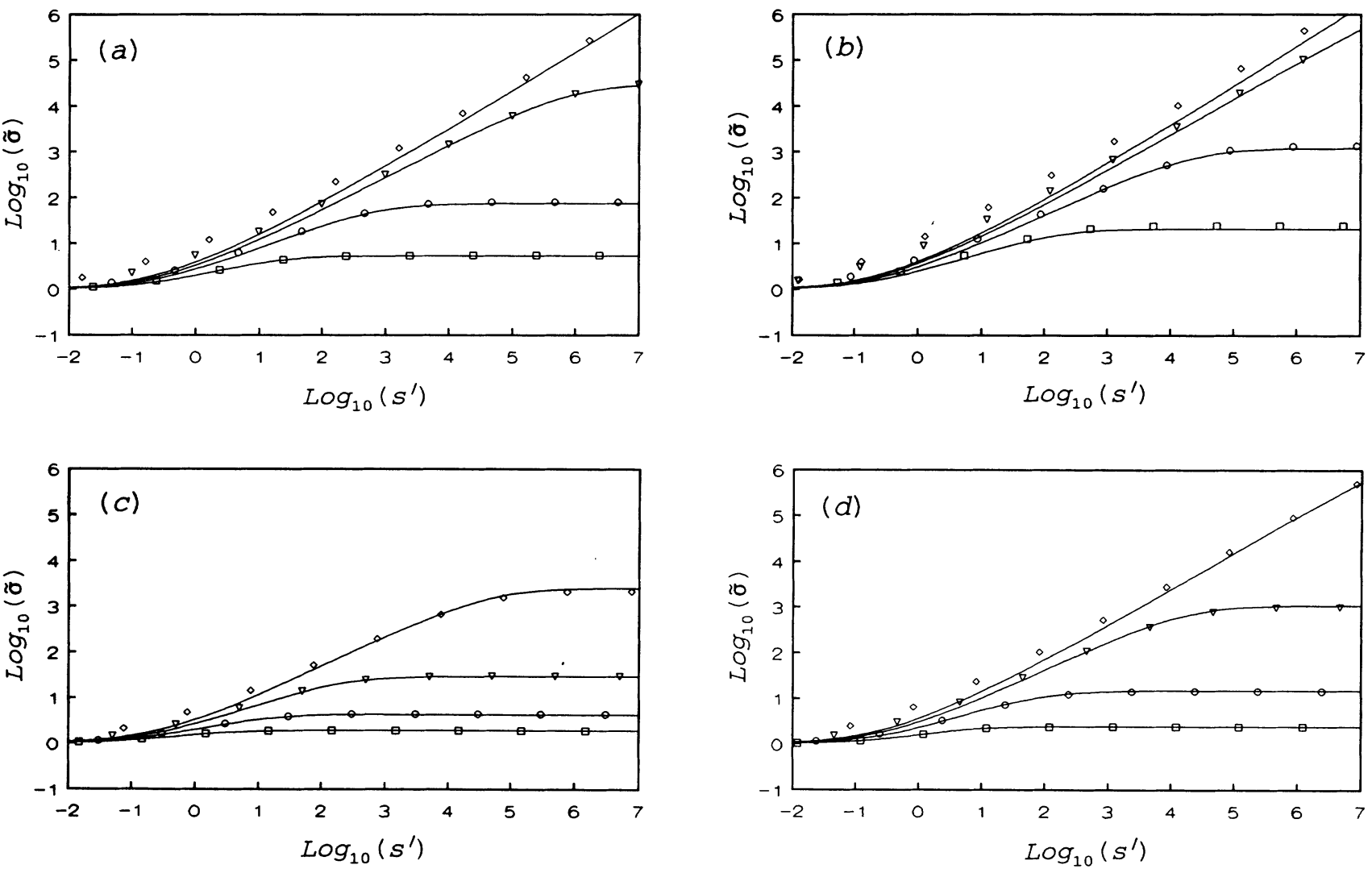

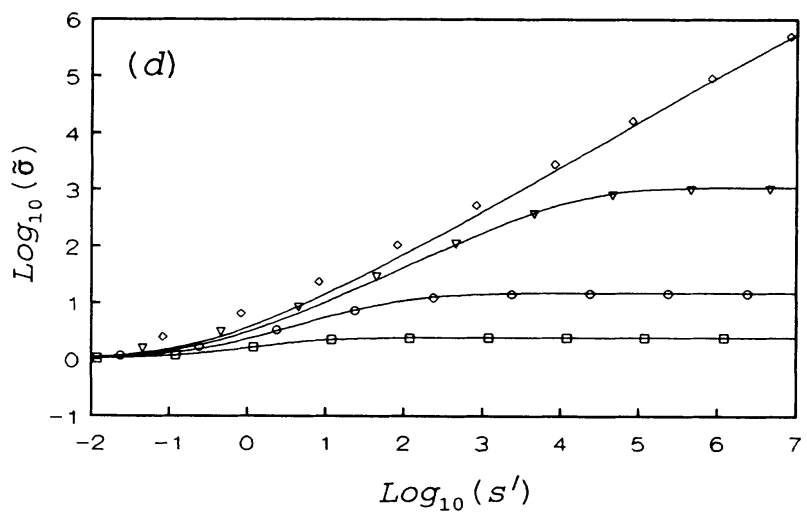

FIG. 4. Log-log plots of the results of computer simulations in two dimensions (points) compared to the EMA predictions (full curves) for symmetric hopping on a $100 \times 100$ lattice. The figures show the conductivity as function of the real Laplace frequency (i.e., at imaginary frequencies). The computer simulations were carried out using the algorithm developed in Sec. III. The points represent averages over 20 different $100 \times 100$ lattices, where the jump frequency activation energy varies according to the following distributions (compare Appendix B of Ref. 20): (a) asymmetric Gaussian; (b) Cauchy; (c) power law with exponent -4 ; and (d) box. Each figure shows the following dimensionless inverse temperatures: $\beta=5(\square), \beta=10(\bigcirc), \beta=20(\nabla)$, and $\beta=40(\diamond)$. The "reduced" Laplace frequency $s^{\prime}$ as well as $\widetilde{\sigma}$ are defined in Eq. (42). The EMA predictions were found by solving Eq. (18) where $s \widetilde{G}$ is given by Eq. (23). 
to average over several simulations to obtain reproducible results.

In each simulation a $100 \times 100$ lattice was generated by, for each link, choosing a random activation energy according to the probability distribution under study. Four different probability distributions were used: The asymmetric Gaussian, the Cauchy, the power law with exponent -4 , and the box. The details of how the energies were generated are described in Appendix B of Ref. 20 ; to avoid spurious correlations the random numbers were generated according to the RANO algorithm of Ref. 51

For each lattice the frequency-dependent conductivity was evaluated from Eq. (40) at a number of frequencies, where the admittance matrix $Y[i, j ; s]$ results from the reduction of the ACMA circuit. For simplicity, the simulations were carried out at real Laplace frequencies, corresponding to purely imaginary frequencies. This trick simplifies the computations and, since $\sigma(s)$ is real for real $s$, makes it possible to present the frequencydependent conductivity in one single curve.

Figure 4 shows a log-log plot of the averages of 20 simulations for the four activation energy distributions at the dimensionless inverse temperatures $\beta=5,10,20$, and 40. If $p(E)$ is the normalized energy probability distribution and one introduces (compare Sec. V)

$$
\widetilde{\beta}=\frac{\beta}{8 \pi p\left(E_{c}\right)},
$$

the quantities $s^{\prime}$ and $\widetilde{\sigma}$ in Fig. 4 are defined by

$$
s^{\prime}=\frac{\widetilde{\beta}}{\sigma(0)} s, \quad \widetilde{\sigma}=\frac{\sigma}{\sigma(0)} .
$$

In Fig. 4 the full curves are the EMA predictions found by solving Eq. (18) numerically. In two dimensions the quantity $s \widetilde{G}$ is given by Eq. (23). A numerical approximation to the elliptic function was used. ${ }^{58}$ Equation (18) was discretized into 10.000 terms and then solved by the bisection method. ${ }^{51}$

The EMA is usually derived from a perturbation expansion around the homogeneous state. As such, there is no a priori reason to believe in the EMA predictions in severely disordered cases like low-temperature hopping, which is really quite extreme: It involves jump frequencies that for $\beta=40$ vary $20-60$ decades. This enormous variation implies that the scaling of the frequency introduced in Eq. (42) shifts the frequency by in some cases more than 15 decades. In this light it must be said that the EMA works rather well in Fig. 4.

\section{THE LOW-TEMPERATURE LIMIT OF THE EMA: THE APPEARANCE OF UNIVERSALITY}

This section studies the EMA prediction for the $T \rightarrow 0$ limit of the frequency-dependent conductivity in symmetric hopping models in more than one dimension. The derivation starts by noting that, as $\beta \rightarrow \infty$,

$$
x=s / \sigma<1
$$

for all frequencies in an increasingly large range around the frequency marking the onset of ac conduction. This observation was first made by Bryksin in a paper from
1980 (Ref. 26) dealing with electrons tunneling between random positions. In the derivation given below, Eq. (43) will be assumed first and subsequently shown to be consistent with the result derived.

Equation (18) is rewritten

$$
0=\left\langle\frac{\Gamma-\sigma}{\Gamma-\sigma+D \sigma /(1-s \widetilde{G})}\right\rangle_{\Gamma} .
$$

When $x$ is small, $s \widetilde{G}$ is small. Expanding to first order in $s \widetilde{G}$ leads to (where the numerator is rewritten for convenience below)

$$
0=\left\langle\frac{\Gamma+[(D-1)+D s \widetilde{G}] \sigma-D(1+s \widetilde{G}) \sigma}{\Gamma+[(D-1)+D s \widetilde{G}] \sigma}\right\rangle_{\Gamma} .
$$

If we introduce the notation $\Gamma(E)=\Gamma_{0} e^{-\beta E}$ to emphasize the activation energy dependence of the jump frequency and if the average is converted into an average over the activation energy probability distribution, Eq. (45) becomes

$$
\frac{1}{D(1+s \widetilde{G}) \sigma}=\left\langle\frac{1}{\Gamma(E)+[(D-1)+D s \widetilde{G}] \sigma}\right\rangle_{E} .
$$

For large $\beta$ the jump frequency $\Gamma(E)$ varies extremely rapidly and, for given $\sigma$ and $s$, there are essentially just two extreme possibilities: Either one has

$$
\Gamma(E) \ll<[(D-1)+D s \widetilde{G}] \sigma
$$

or the opposite extreme. In the former case $\Gamma(E)$ may be ignored altogether from the denominator in Eq. (46), while in the latter case the denominator becomes very large and there is little contribution to the right-hand side. The energy separating the two cases, $E_{g}(s)$, is given as the solution of

$$
\Gamma(E)=[(D-1)+D s \widetilde{G}] \sigma ;
$$

thus

$$
E_{g}(s)=\frac{-1}{\beta} \ln \left[\frac{[(D-1)+D s \widetilde{G}] \sigma}{\Gamma_{0}}\right] .
$$

Accordingly, the right-hand side of Eq. (46) becomes

$$
\begin{aligned}
& \left\langle\frac{1}{\Gamma(E)+[(D-1)+D s \widetilde{G}] \sigma}\right\rangle_{E} \\
& =\frac{1}{[(D-1)+D s \widetilde{G}] \sigma} \int_{E_{g}(s)}^{\infty} p(E) d E
\end{aligned}
$$

and Eq. (46) reduces to

$$
\frac{D-1}{D}+\frac{s \widetilde{G}}{D(1+s \widetilde{G})}=\int_{E_{g}(s)}^{\infty} p(E) d E .
$$

Evaluating Eq. (49) at $s=0$ gives an expression for $(D-1) / D$. When this expression is subtracted from Eq. (49), one gets

$$
\frac{s \widetilde{G}}{D(1+s \widetilde{G})}=\int_{E_{g}(s)}^{E_{g}(0)} p(E) d E .
$$

For large $\beta, E_{g}(0)$ is close to $E_{g}(s)$ and therefore the integral on the right-hand side can easily be evaluated. If $p\left[E_{g}(0)\right]$ is denoted by $p_{0}$, the integral is simply 
$p_{0}\left[E_{g}(0)-E_{g}(s)\right]$. Introducing the symbol $\widetilde{\sigma}=\sigma / \sigma(0)$ into Eq. (50) and using Eq. (47) one gets

$$
\frac{s \widetilde{G}}{D(1+s \widetilde{G})}=\frac{p_{0}}{\beta} \ln \left[\left(1+\frac{D}{D-1} s \widetilde{G}\right) \widetilde{\sigma}\right] \text {. }
$$

To leading order in the small quantity $s \widetilde{G}$, Eq. (51) reduces to

$$
\frac{\beta}{D p_{0}} s \widetilde{G}=\ln [\widetilde{\sigma}]
$$

Since for $D=1$ one has $E_{g}(0)=\infty$, the derivation assumes $D>1$. As $\beta \rightarrow \infty, E_{g}(0)$ approaches the dc conductivity activation energy which is equal to the percolation energy defined by Eq. (17); thus

$$
p_{0}=p\left(E_{c}\right) \text {. }
$$

In further development one has to distinguish between the cases $D=2$ and $D>2$. In the latter case, which is the simplest, the quantity $s \widetilde{G}$ as a function of $x=s / \sigma$ contains a regular first-order term. Writing

$$
D>2: \quad s \widetilde{G}=\alpha_{D} x \quad(x \rightarrow 0),
$$

Eq. (19) implies

$\alpha_{D}=\frac{1}{2} \int_{1-B Z} \frac{d^{D} k}{(2 \pi)^{D}} \frac{1}{D-\left(\cos k_{1}+\cdots+\cos k_{D}\right)}$.

For $D=3$ one has $\alpha_{3}=0.253$. $^{59}$ Substituting the expansion Eq. (54) into Eq. (52) leads to Eq. (1), $\widetilde{\sigma} \ln (\widetilde{\sigma})=\widetilde{s}$, where

$$
D>2: \quad \widetilde{s}=\frac{\beta \alpha_{D}}{D p_{0} \sigma(0)} s .
$$

Finally, the consistency of the derivation is checked: The assumption Eq. (43) is indeed satisfied, since for $\beta \rightarrow \infty$ one has $x=s / \sigma \propto \widetilde{s} /(\widetilde{\sigma} \beta) \rightarrow 0$ for fixed $\widetilde{\sigma}$ and $\widetilde{s}$.

Turning now to the two-dimensional case [where the integral in Eq. (55) diverges] we use the asymptotic expansion of the complete elliptic integral of the first kind: ${ }^{60}$ For $k \rightarrow 1$ one has $K(k)=\ln \left(4 / k^{\prime}\right)$ where $k^{2}+k^{\prime 2}=1$. This implies that

$$
K(k)=\frac{-1}{2} \ln (1-k)
$$

for $k \rightarrow 1$. Thus,

$$
K\left(\frac{4}{4+x}\right)=\frac{-1}{2} \ln (x)
$$

for $x \rightarrow 0$. When this is substituted into Eq. (23) one finds asymptotically

$$
s \widetilde{G}_{2-D}=\frac{-1}{4 \pi} x \ln (x) \quad(x \rightarrow 0) .
$$

In terms of the $\widetilde{\beta}$ defined in Eq. (41), Eq. (52) thus becomes

$$
\ln (\widetilde{\sigma})=\widetilde{\beta} \frac{s}{\sigma} \ln \left(\frac{\sigma}{s}\right) .
$$

Defining now

$$
D=2: \quad \widetilde{s}=\frac{\widetilde{\beta} \ln (\widetilde{\beta})}{\sigma(0)} s,
$$

Eq. (58) becomes

$\ln (\widetilde{\sigma})=\widetilde{\beta} \frac{1}{\widetilde{\sigma}} \frac{\widetilde{s}}{\widetilde{\beta} \ln (\widetilde{\beta})}\{\ln (\widetilde{\sigma})-\ln (\widetilde{S})+\ln (\widetilde{\beta})+\ln [\ln (\widetilde{\beta})]\}$.

For fixed $\widetilde{\sigma}$ and $\widetilde{s}$ as $\widetilde{\beta} \rightarrow \infty$, Eq. (60) reduces to Eq. (1), $\widetilde{\sigma} \ln (\widetilde{\sigma})=\widetilde{s}$. Note that the assumption $x=s / \sigma<<1$ is also satisfied for $D=2$ for fixed $\widetilde{\sigma}$ and $\widetilde{s}$ when $\widetilde{\beta}$ is sufficiently large.

The numerical solution of Eq. (1) was discussed in Appendix $A$ in Ref. 20 where an analytical approximation to the function $\widetilde{\sigma}(\widetilde{S})$ was also given.

Figure 5 tests the EMA universality prediction against computer simulations. The four different activation energy distributions of Fig. 4 were used, supplemented by results for the exponential distribution. ${ }^{20}$ For each distribution the temperature was chosen so that $\widetilde{\beta}=4$. Each point in the figure represents the average of 50 simulations of a $100 \times 100$ lattice. The figure clearly shows that there is universality at low temperatures, but there is not a quantitative agreement with the EMA universality prediction. A further discussion of this result is given in the next section.

\section{DISCUSSION}

In this paper a method for the numerical solution of symmetric hopping models has been derived. The method, which makes use of the ac Miller Abrahams

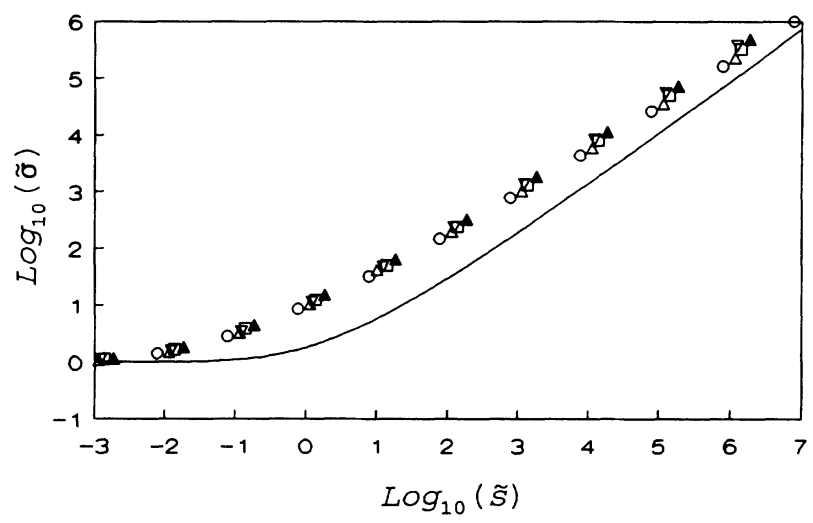

FIG. 5. Log-lot plot comparing the EMA universality prediction [full curve; Eq. (1)] to computer simulations (points) of a $100 \times 100$ lattice for five different activation energy probability distributions. Each point represents the average of 50 simulations taken at the "reduced" inverse dimensionless temperature $\widetilde{\beta}=4$ where $\widetilde{\beta}$ is defined in Eq. (41); this corresponds to (the relevant $E_{c}$ 's are given in Appendix B of Ref. 20) $\beta=63.91$ for the asymmetric Gaussian, $\beta=32.00$ for the Cauchy, $\beta=50.27$ for the exponential, $\beta=119.66$ for the power law with exponent -4 , and $\beta=100.53$ for the box distribution. The "reduced" Laplace frequency $\widetilde{S}$ is defined in Eq. (59) and $\widetilde{\sigma}=\sigma / \sigma(0)$. The figure shows results for the following distributions: asymmetric Gaussian ( $\Delta$ ); Cauchy (O); exponential $(\square)$; power law with exponent $-4(\triangle)$; and box $(\nabla)$. 
electrical equivalent circuit, may be generalized immediately to deal with nonsymmetric hopping models; the only change is that in the ACMA circuit the capacitors then vary from site to site. ${ }^{14,54}$ The method allows a faster and more accurate calculation of $\sigma(s)$ in hopping models for larger lattices and at lower temperatures than previous methods. Thus, the standard Monte Carlo type method is useless if one wants to study low temperatures as in Fig. 4. The standard relaxation methods for solving linear equations are also too slow in this regime where the coefficients vary many orders of magnitude.

There exists a clever algorithm for solving linear equations with coefficients that vary many orders of magnitude. This is the algebraic multigrid algorithm $(A M G)^{61,62}$ which is available from the Yale multigrid library in a well-documented and carefully debugged FORTRAN version. ${ }^{63}$ The AMG is an algebraic generalization of the multigrid method for solving elliptic partial differential equations. The AMG has been tested successfully for large random admittance networks with admittances varying many orders of magnitude. ${ }^{20,64}$ It solves the Kirchhoff equations in a time proportional to the number of equations. For the present problem, the AMG solves the problem in $D$ dimensions in a time $\propto N^{D}$. At first sight, this is much better than the method presented in Sec. III, which, as is easy to show for $D>1$, calculates the conductivity in a time $\propto N^{3 D-2}$. However, in the practical use of the AMG it is not presently superior to the method of Sec. III. Thus, when applied to a hopping problem at low temperatures, the AMG easily runs into overflow problems, whereas the method of Sec. III avoids such problems. At low temperatures, if one wants to calculate the conductivity by solving Eq. (26) or the higherdimensional analogs, the solution must be extremely accurate. The standard double precision real number representation is not enough, since the equations should be solved with an accuracy of 50-100 digits (depending on how low the temperature is). Unfortunately, higher precisions are not hardware implemented today and are therefore quite slow. The method presented in Sec. III seems to be the best available at present. On a longer time span it is likely that the AMG will eventually become the best choice.

The results of extensive computer simulations of a $100 \times 100$ lattice in 2D was reported in Sec. IV. In order to obtain reproducible results at low temperatures, it was necessary to average over several simulations of different lattices generated for the same activation energy probability distribution. The main problem in the reproducibility lies at low frequencies; thus, at frequencies where $\log _{10}(\widetilde{\sigma})>1$ the results (i.e., $\widetilde{\sigma}$ as function of $\left.s^{\prime}\right)$ are generally quite reproducible.

The results of the computer simulations were compared to the predictions of the EMA at real Laplace frequencies at a number of temperatures in Fig. 4. The use of real Laplace frequencies not only simplifies the calculations, but also makes it possible to represent the results in one curve (instead of two, one for the real part and one for the imaginary part of the conductivity). This curve contains all information about the frequency dependence of the conductivity. This is because the function $\sigma(s)$ is analytic in the upper half $s$ plane so, by analytic continuation, the behavior on the real $s$ axis determines the function uniquely. A further virtue of this representation is that deviations from the EMA are somewhat magnified here compared to the use of real frequencies. The "reduced" frequency used in Fig. 4 is not the $\widetilde{s}$ of Eq. (59) simply because, for some of the highest temperatures studied, $\widetilde{\beta}$ becomes less than one, so that Eq. (59) does not make sense. Instead, the related "reduced" frequency $s^{\prime}=\widetilde{\beta} s / \sigma(0)[\mathrm{Eq} .(42)]$ was used in Fig. 4.

As far as is known to the author, these results are the first simulations of a hopping model at low temperatures where the jump frequencies $\Gamma$ vary over several decades (here up to about 50-60 decades). In general, there is a rather good agreement between the simulations and the EMA, with some deviations in the transition region where the EMA at low temperatures consistently underestimates the conductivity.

At low temperatures the EMA predicts a universal frequency dependence of the conductivity, independent of the activation energy probability distribution (Sec. V). A particular case of the equation governing the universal conductivity, Eq. (1) was derived by Bryksin in 1980 (Ref. 26) for a system of tunneling electrons. The equation was later derived by Fishchuk ${ }^{24}$ for the box distribution of activation energies in the macroscopic model, and by the present author for a hopping model with the box distribution. ${ }^{30}$ Recently, it was shown by means of the EMA for random admittance networks that Eq. (1) is universal in the low-temperature limit of the macroscopic model. ${ }^{19,20}$ In Sec. $V$ it was shown that the equation is also the universal EMA prediction for symmetric hopping models. Physically what happens is that, at low temperatures, the conduction mainly follows the percolation paths, and the only "signature" of the activation energy distribution left is the number $p\left(E_{c}\right)$.

The universality prediction was tested in Fig. 5, which studies five different activation energy probability distributions at the same "reduced" temperature $\widetilde{\beta}=4$. There is clearly a universality in the sense that the function $\widetilde{\sigma}(\widetilde{S})$ is independent of the activation energy distribution. However, the results deviate from the EMA prediction Eq. (1). One reason for the discrepancy may be that the temperature is simply not low enough in Fig. 5. Unfortunately, it is not possible to go to lower temperatures for a $100 \times 100$ lattice without loosing reproducibility.

There are interesting differences between the macroscopic model ${ }^{20}$ and hopping models. Figures 4 and 5 indicate a systematic deviation of the simulations from the EMA predictions at low temperatures in the transition region. Here the data give a less sharp transition to frequency dependence than the EMA predicts. In the macroscopic model, on the other hand, there is a very good agreement between the EMA predictions and the simulations at all temperatures and frequencies. It is not clear what the origin of these differences is. Apparently, the hopping model is more complex than the macroscopic model. Thus, in the derivation of universality for hopping models, one has to distinguish between the cases $D=2$ and $D>2$, which is not necessary for the derivation of Eq. (1) for the macroscopic model. ${ }^{20}$ 
In the simplest realistic approximation to hopping models, the continuous time random walk (CTRW) approximation, ${ }^{35}$ the conductivity for the box distribution of energy barriers ${ }^{30}$ is

$$
\widetilde{\sigma}=\frac{\widetilde{s}}{\ln (1+\widetilde{s})} .
$$

As has been shown elsewhere ${ }^{20}$ this expression is close to that given by Eq. (1); in particular, the two functions $\widetilde{\sigma}(\widetilde{s})$ have the same asymptotic expressions for the exponents of the real and imaginary parts of the conductivity. These exponents converge slowly to one as the frequency goes to infinity ${ }^{20}$ (A convincing experimental demonstration of this phenomenon has recently been given for metal-cluster compounds. ${ }^{8}$ )

There has been relatively little discussion of universality for ac conduction in the literature. ${ }^{1-3,8}$ In experiments, a number of authors pointed out early that quite different systems like ionic conductive glasses and amorphous semiconductors have surprisingly similar ac responses. $^{1-3}$ In a theoretical paper, Summerfield ${ }^{65}$ in 1985 termed the phrase "quasi-universality" for the fact that a number of different models, when solved in the EPA,${ }^{54}$ give almost the same frequency dependence of the conductivity.

The macroscopic model based on Maxwell's equations for an inhomogeneous solid leads to an electrical equivalent circuit, where the nodes on a cubic lattice are joined by a capacitor and a resistor in parallel. ${ }^{20,24}$ The resistors carry the free charge currents while the capacitor currents are Maxwell's displacement currents. ${ }^{20}$ In contrast to the circuit of Fig. 2, there are no connections to the ground and no voltage generators; the "solid" is simply subjected to a macroscopic potential drop at the electrodes (equal to two opposing end faces). In the dc limit the macroscopic model and hopping models both give simple resistance circuits. Thus, the dc limit of the EMA hopping equation, Eq. (21), is identical to the EMA equation for a random resistance circuit.

An interesting connection between the symmetric hopping model and the macroscopic model is that the CTRW approximation ${ }^{28,35}$ to hopping models corre- sponds to the one-dimensional version of the macroscopic model. As has been discussed in detail elsewhere, ${ }^{20}$ this version becomes realistic at low temperatures where conduction mainly follows the percolation paths. Along these lines, an approximation to the macroscopic model referred to as the "percolation path approximation" (PPA) was proposed in Ref. 20, leading to Eq. (61).

Throughout this paper the limit of extreme disorder (where the jump frequencies $\Gamma$ vary several decades) was arrived at by going to low temperatures for a system with thermally activated hopping. The same limit is also arrived at in a system of localized electrons tunneling between nearest-neighbor sites, when the density of electrons becomes very small. The system of tunneling electrons has been studied extensively in the past. ${ }^{26,54,66}$ Though it was not spelled out in detail in Sec. V, in the extreme disorder limit, the EMA universality prediction [Eq. (1)] applies to this system as well. ${ }^{26}$

There are a number of open problems and work that remains to be done. The symmetric hopping model should be studied numerically at low temperatures also in three dimensions. Regarding the numerical method, it is not clear what the optimal strategy for removing nodes is. From the theoretical point of view the main question is: Is there true universality in the extreme disorder limit, or is there only "quasi-universality?" If true universality does exist, as believed by the author, is the universal function $\widetilde{\sigma}(\widetilde{s})$ the same in all dimensions? If this is not the case, analytical methods more accurate than the EMA should be developed to calculate the universal conductivity. A further question is: What is the cause of the difference between the hopping model and the macroscopic model, where the EMA works better than for hopping?

\section{ACKNOWLEDGMENTS}

The author wishes to thank P. Borgstrom, H. Larsen, I. H. Pedersen, and T. Riedel for technical assistance. This work was supported by the Danish Natural Science Research Council.
${ }^{1}$ A. K. Jonscher, Nature 267, 673 (1977); Dielectric Relaxation in Solids (Chelsea Dielectric, London, 1983).

${ }^{2}$ A. E. Owen, J. Non-Cryst. Solids 25, 372 (1977).

${ }^{3}$ A. Mansingh, Bull. Mater. Sci. (India) 2, 325 (1980).

${ }^{4}$ A. R. Long, Adv. Phys. 31, 553 (1982).

${ }^{5}$ M. D. Ingram, Phys. Chem. Glasses 28, 215 (1987).

6 S. R. Elliott, Physics of Amorphous Materials, 2nd ed. (Longman Scientific, London, 1990).

${ }^{7}$ C. A. Angell, Chem. Rev. 90, 523 (1990).

${ }^{8}$ M. P. J. van Staveren, H. B. Brom, and L. J. de Jongh, Phys. Rep. 208, 1 (1991).

9J. L. Barton, Verres Réfract. 20, 328 (1966).

${ }^{10} \mathrm{~T}$. Nakajima, in 1971 Annual Report, Conference on Electric Insulation and Dielectric Phenomena (National Academy of Sciences, Washington, DC, 1972), p. 168.

${ }^{11}$ H. Namikawa, J. Non-Cryst. Solids 18, 173 (1975).
${ }^{12} \mathrm{M}$. Tomozawa, in Treatise on Materials Science, Vol. 12, edited by M. Tomozawa (Academic, New York, 1977), p. 283.

${ }^{13}$ J. C. Dyre, J. Non-Cryst. Solids 88, 271 (1986).

${ }^{14}$ H. Böttger and V. V. Bryksin, Hopping Conduction in Solids (Akademie-Verlag, Berlin, 1985).

${ }^{15}$ J. W. Haus and K. W. Kehr, Phys. Rep. 150, 263 (1987).

16J.-P. Bouchaud and A. Georges, Phys. Rep. 195, 127 (1990).

${ }^{17}$ J. O. Isard, Philos. Mag. B 62, 139 (1990); Philos. Mag. A 66, 213 (1992).

${ }^{18}$ P. Maass, J. Petersen, A. Bunde, W. Dieterich, and H. E. Roman, Phys. Rev. Lett. 66, 52 (1991).

19J. C. Dyre, Phys. Rev. B 47, 9128 (1993).

${ }^{20}$ J. C. Dyre, Phys. Rev. B 48, 12511 (1993).

${ }^{21}$ B. E. Springett, Phys. Rev. Lett. 31, 1463 (1973).

${ }^{22}$ I. Webman, J. Jortner, and M. H. Cohen, Phys. Rev. B 15, 5712 (1977). 
23J. Sinkkonen, Phys. Status Solidi B 103, 231 (1981).

${ }^{24}$ I. I. Fishchuk, Phys. Status Solidi A 93, 675 (1986).

${ }^{25}$ S. Kirkpatrick, Rev. Mod. Phys. 45, 574 (1973).

${ }^{26}$ V. V. Bryksin, Fiz. Tverd. Tela (Leningrad) 22, 2441 (1980) [Sov. Phys. Solid State 22, 1421 (1980)].

${ }^{27} \mathbf{P}$. N. Butcher, in Linear and Nonlinear Electronic Transport in Solids, edited by J. T. de Vreese and V. E. van Doren (Plenum, New York, 1976), p. 341.

${ }^{28}$ M. Lax and T. Odagaki, in Macroscopic Properties of Disordered Media, edited by R. Burridge (Springer-Verlag, Berlin, 1982), p. 148.

${ }^{29}$ G. A. Niklasson, J. Appl. Phys. 62, R1 (1987).

30J. C. Dyre, J. Appl. Phys. 64, 2456 (1988).

${ }^{31}$ H. Haken, Synergetics (Springer, Berlin, 1983).

${ }^{32}$ N. G. van Kampen, Stochastic Processes in Physics and Chemistry (North-Holland, Amsterdam, 1981).

${ }^{33}$ B. I. Shklovskii and A. L. Efros, Electronic Properties of Doped Semiconductors (Springer-Verlag, Berlin, 1984).

${ }^{34}$ R. Kubo, J. Phys. Soc. Jpn. 12, 570 (1957).

${ }^{35}$ H. Scher and M. Lax, Phys. Rev. B 7, 4491 (1973); 7, 4502 (1973).

${ }^{36}$ S. Alexander, J. Bernasconi, W. R. Schneider, and R. Orbach, Rev. Mod. Phys. 53, 175 (1981).

${ }^{37}$ J. C. Kimball and L. W. Adams, Phys. Rev. B 18, 5851 (1978).

${ }^{38}$ V. Ambegaokar, B. I. Halperin, and J. S. Langer, Phys. Rev. B 4, 2612 (1971).

${ }^{39}$ B. I. Shklovskii and A. L. Efros, Zh. Eksp. Teor. Fiz. 60, 867 (1971) [Sov. Phys. JETP 33, 468 (1971)].

${ }^{40}$ S. Tyc and B. I. Halperin, Phys. Rev. B 39, 877 (1989).

${ }^{41}$ H. Kesten, Percolation Theory for Mathematicians (Birkhauser, Boston, 1982).

${ }^{42}$ D. B. Gingold and C. J. Lobb, Phys. Rev. B 42, 8220 (1990).

${ }^{43}$ J. A. Krumhansl, in Amorphous Magnetism, edited by H. O. Hooper and A. M. de Graaf (Plenum, New York, 1973), p. 15.

${ }^{44} \mathrm{~F}$. Yonezawa, in The Structure and Properties of Matter, edited by T. Matsubara (Springer-Verlag, Berlin, 1982), p. 383.

${ }^{45}$ S. Summerfield, Solid State Commun. 39, 401 (1981).
${ }^{46}$ B. Movaghar and W. Schirmacher, J. Phys. C 14, 859 (1981).

${ }^{47}$ T. Odagaki and M. Lax, Phys. Rev. B 24, 5284 (1981).

${ }^{48}$ W. Schirmacher, Ber. Bunsenges. Phys. Chem. 95, 368 (1991).

${ }^{49}$ E. N. Economou, Green's Functions in Quantum Physics (Springer, Berlin, 1983).

${ }^{50}$ M. Sahimi, B. D. Hughes, L. E. Scriven, and H. T. Davis, J. Chem. Phys. 78, 6849 (1983).

${ }^{51}$ W. H. Press, B. P. Flannery, S. A. Teukolsky, and W. T. Vetterling, Numerical Recipes (Cambridge University Press, Cambridge, 1986).

${ }^{52}$ A. Miller and B. Abrahams, Phys. Rev. 120, 745 (1960).

${ }^{53} \mathrm{M}$. Pollak, in Proceedings of the Fifth International Conference on Amorphous and Liquid Semiconductors, GarmischPartenkirchen 1973, Vol. 1 (Taylor and Francis, London, 1974), p. 127.

${ }^{54}$ S. Summerfield and P. N. Butcher, J. Phys. C 15, 7003 (1982).

${ }^{55}$ N. M. Morris, Electrical Circuit Analysis and Design (Macmillan, London, 1993).

${ }^{56}$ R. Fogelholm, J. Phys. C 13, L571 (1980).

${ }^{57}$ R. Fogelholm, Report No. TRITA-FYS-5075, Royal Institute of Technology, Stockholm, 1980 (unpublished).

${ }^{58}$ Handbook of Mathematical Functions, edited by $\mathbf{M}$. Abramowitz and I. A. Stegun (Dover, New York, 1965).

${ }^{59}$ M. Lax, Phys. Rev. 97, 629 (1955).

${ }^{60}$ H. B. Dwight, Tables of Integrals and Other Mathematical Data (Macmillan, New York, 1961).

${ }^{61}$ K. Stuben, Appl. Math. Comput. 13, 419 (1983).

${ }^{62}$ A. Brandt, Appl. Math. Comput. 19, 23 (1986).

${ }^{63}$ The AMG1R5 FORTRAN subroutine is available from "casper.na.cs.yale.edu" (128.36.12.1); ftp as anonymous, then look for the file "amg.tar.Z" in the "gmd" directory under the "mgnet" directory.

${ }^{64}$ R. G. Edwards, J. Goodman, and A. D. Sokal, Phys. Rev. Lett. 61, 1333 (1988).

${ }^{65}$ S. Summerfield, Philos. Mag. B 52, 9 (1985).

${ }^{66}$ B. Movaghar and W. Schirmacher, J. Phys. C 14, 859 (1981). 